\title{
Using Andersen's model of health care utilization to assess factors associated with COVID-19 testing among adults in nine low-and middle-income countries: an online
}

\section{survey}

Supa Pengid ${ }^{1,2}$, Karl Peltzer ${ }^{2,3 *}$, Edlaine Faria de Moura Villela ${ }^{4,5}$, Joseph Nelson Siewe Fodjo ${ }^{6}$, Ching Sin Siau ${ }^{7}$, Won Sun Chen ${ }^{8}$, Suzanna A. Bono ${ }^{9}$, Isareethika Jayasvasti ${ }^{10}$, M. Tasdik Hasan ${ }^{11,12,13}$, Rhoda K. Wanyenze ${ }^{14}$, Mina C. Hosseinipour ${ }^{15}$, Housseini Dolo ${ }^{16,17}$, Philippe Sessou ${ }^{18}$, John D. Ditekemena ${ }^{19}$ and Robert Colebunders ${ }^{6}$

\begin{abstract}
Background: This study aimed to investigate, using Andersen's model of health care utilization, factors associated with COVID-19 testing among adults in nine low- and middle- income countries.

Methods: In between 10 December 2020 and 9 February 2021, an online survey was organized in nine lowand middle-income countries. In total 10,183 adults (median age 45 years, interquartile range 33-57years, range 18-93 years), including 6470 from Brazil, 1738 Malaysia, 1124 Thailand, 230 Bangladesh, 219 DR Congo, 159 Benin, 107 Uganda, 81 Malawi and 55 from Mali participated in the study. COVID-19 testing/infection status was assessed by self-report.
\end{abstract}

Results: Of the 10,183 participants, 40.3\% had ever tested for COVID-19, 7.3\% tested positive, and 33.0\% tested negative. In an adjusted logistic regression model, predisposing factors (residing in Brazil, postgraduate education), enabling/disabling factors (urban residence, higher perceived economic status, being a student or worker in the health care sector, and moderate or severe psychological distress), and need factors (having at least one chronic condition) increased the odds of COVID-19 testing. Among those who were tested, participants residing in Bangladesh, those who had moderate to severe psychological distress were positively associated with COVID-19 positive diagnosis. Participants who are residing in Malaysia and Thailand, and those who had higher education were negatively associated with a COVID-19 positive diagnosis. Considering all participants, higher perceived economic status, being a student or worker in the health sector, and moderate or severe psychological distress were positively associated with a COVID-19 positive diagnosis, and residing in Malaysia, Thailand or five African countries was negatively associated with a COVID19 positive diagnosis.

*Correspondence: kfpeltzer@gmail.com

${ }^{3}$ Department of Psychology, College of Medical and Health Science, Asia

University, Taichung, Taiwan

Full list of author information is available at the end of the article original author(s) and the source, provide a link to the Creative Commons licence, and indicate if changes were made. The images or other third party material in this article are included in the article's Creative Commons licence, unless indicated otherwise in a credit line to the material. If material is not included in the article's Creative Commons licence and your intended use is not permitted by statutory regulation or exceeds the permitted use, you will need to obtain permission directly from the copyright holder. To view a copy of this licence, visit http://creativecommons.org/licenses/by/4.0/. The Creative Commons Public Domain Dedication waiver (http://creativeco mmons.org/publicdomain/zero/1.0/) applies to the data made available in this article, unless otherwise stated in a credit line to the data. 
Conclusion: A high rate of COVID-19 testing among adults was reported in nine low-and middle-income countries. However, access to testing needs to be increased in Africa. Moreover, COVID-19 testing programmes need to target persons of lower economic status and education level who are less tested but most at risk for COVID-19 infection.

Keywords: COVID-19, testing, adults, LMICS

\section{Background}

In places with ongoing COVID-19 transmission, mass testing (including both symptomatic and asymptomatic individuals) can "help prevent the spread of COVID-19 and identify people in need of care in a timely fashion. A positive test early in the course of the illness enables individuals to isolate themselves thereby reducing the chances of infecting others and allowing them to seek treatment earlier, likely reducing disease severity and the risk of long-term disability, or death." [1]. A negative test on the other hand does not exempt one from possibly acquiring and transmitting the infection in the future [1]. Therefore, even if an individual is tested negative at a given time point, continuation of safe hygienic measures (washing hands with soaps/using sanitizers frequently, physical distancing, and wearing a face mask) is recommended to protect self and others from COVID-19 [1]. A positive test means isolation is mandatory, and that others with whom the individual may have been in contact since the time of exposure should also get tested [1]. Since nearly half of all SARS-CoV-2 infections are transmitted by pre-symptomatic and asymptomatic people, early identification of infected individuals will play a major role in containing the pandemic [1].

Scanty research has been conducted to identify factors associated with the uptake of COVID-19 testing. In a cross-sectional population-wide survey in Ontario, Canada, of the 758,691 participants who had been tested, $3.3 \%$ tested positive for SARS-CoV-2 [2]. The odds of being tested increased with age, male sex, several underlying health conditions, previous use of health care services, and higher household income [2]. Comparing the odds of COVID-19 positive diagnosis, vs having a negative test or being untested, older age, certain comorbidities, such as hypertension, diabetes and heart disease, and higher previous use of health care services were associated with increased odds of a positive SARS-CoV-2 test result [2]. Using UK biobank data, being tested for COVID-19 was associated with male sex, Black ethnicity, lower socioeconomic status, occupation (being a health care worker, unemployed, or retired), comorbidities, exposure to particulate matter (PM) 2.5 absorbance, and ever smoker [3]. Among the tested individuals, only non-White ethnicity, male sex, and lower education were associated with a COVID-19 positive diagnosis [3]. Regarding COVID-19 test uptake/outcome, another important factor to consider is the fact that workers in the healthcare sector stand a higher risk of testing positive. Indeed, a meta-analytic study showed that the pooled prevalence of healthcare workers testing positive for COVID-19 was between 7 to $11 \%$ [4], compared to 0.46 to $1.82 \%$ in a German population-based cohort study [5]. In a web survey in the Rio de Janeiro metropolitan area, Brazil, in 2020, 42.6\% reported ever testing for COVID-19, and the prevalence of a positive PCR results was $16.0 \%$, and of a positive antibody result $10.0 \%$, with no difference across age and comorbidity groups [6].

Health care utilization in terms of COVID-19 testing uptake can be assessed using the Andersen behavioural model explaining that health care utilization is determined by predisposing, enabling/disabling, and needing factors [7]. Predisposing (demographic and social) factors reflect the individuals' propensity to use health services, enabling/disabling (economic, knowledge) factors are the resources that may facilitate access to services, and the need (health outcome) factors represent potential needs of health service use, such as perceived health status, and chronic conditions $[8,9]$.

In the current study, we aimed to investigate, using Andersen's model of health care utilization, factors associated with COVID-19 testing among adults in nine low- and middle- income countries (LMICs) in different phases of the pandemic. Participating countries included Brazil from South America, Malaysia, Bangladesh and Thailand from Southeast Asia and the Democratic Republic of the Congo as the main country from Africa. In nationwide COVID-19 seroprevalence surveys in Brazil the prevalence was $1.9 \%$ in May 2020 and 3.1\% in June 2020 [10]. In Malaysia, between 16 March 2020 and 31 May 2021, Malaysia's average 7-day incidence rate was 26.6 reported infections per 100,000 population, and the average test positive ratio and testing rate were $4.3 \%$ and 0.8 tests per 1000 population [11]. In a cross-sectional online survey in 2020 in Bangladesh, 315 (15.1\%) of 2080 individuals reported to have experienced a COVID-19 infection [12], and in a retrospective cohort study of adult hospitalized patients in 2020 in Thailand, 107 (7.5\%) of 1409 patients were COVID-19 infected [13]. In a crosssectional survey in the general population in Kinshasa, Democratic Republic of the Congo, in 2020, the overall weighted, age-standardized SARS-CoV-2 seroprevalence was $16.6 \%$, and the estimated infection-to-case ratio 
was 292:1 [14]. Cumulative COVID-19 tests performed per 1000 people were in Brazil 142.47, Malaysia 177.78, Thailand 31.25, Bangladesh 21.12, Uganda 17.27, and Malawi 6.76 on 22 January 2021 [15]. The total number of COVID-19 tests per new confirmed case were on 3 February 2021 in Thailand 111.1, Malaysia 30.3, Bangladesh 6.9, and Malawi 5.7 [16]. COVID-19 vaccine doses administered per 100 people was in Brazil 0.06 on 21 January 2021 [17]. Lack of COVID-19 testing capacity, barriers in accessing COVID-19 testing because of high direct and indirect costs, and stigma associated with COVID19 testing contribute to insufficient COVID-19 testing in LMICs $[18,19]$.

\section{Methods}

\section{Study design, sample, and procedure}

This was a descriptive cross-sectional online study conducted in nine LMIC between 10 December 2020 to 9 February 2021. This study was organised by the International Citizen Project (ICP) COVID-19 (ICPCovid) to monitor adherence to COVID-19 prevention measures in low- and middle-income countries [20]. Study countries were selected based on their willingness to participate in the ICP; 50 participants per country was the minimum requirement for enough statistical power [21]. Participant inclusion criteria were being aged 18 years and older, any gender, and provision of electronic informed consent, and exclusion criteria were younger than 18 years and not providing electronic informed consent. Ethical approval was obtained from the Ethics Committees of the participating countries. Further details of the research procedures have been described previously [22]. Briefly, a pre-tested online questionnaire was disseminated via various platforms and consenting respondents submitted their responses via computer/smartphones; all the collected information was stored in a secure server until data extraction and analysis.

\section{Measures}

Using Andersen's model of health care utilization [7], study variables were categorized into outcome variable, predisposing factors, enabling/disabling factors, and need for care factors.

\section{Outcome variable}

COVID-19 testing/infection status was assessed with the question, "Since the beginning of the COVID-19 outbreak, do you have information on your infection status?" Response options were $1=$ not tested/does not know test results, $2=$ negative, and $3=$ positive.

\section{Predisposing factors}

Sociodemographic factors included age (number), sex (male, female), country of residence, educational level (primary, secondary, university undergraduate degree holder, university postgraduate degree holder) and the (estimated) age(s) of their housemate(s) (number: $<12$ years, $12-17$ years, $18-59$ years, and $\geq 60$ years).

\section{COVID-19 preventive measures}

Participants were asked, "During the past 7 days, have you been observing any of the following preventive measures against COVID-19? 1) Social distancing of at least $1.5 \mathrm{~m}, 2$ ) Wearing a face mask, 3) Hand hygiene (regular handwashing with soap or using hand gel), and 4) Coughing hygiene (covering the mouth when coughing or sneezing) (Yes/No). A composite non-adherence to all four COVID-19 preventive measure was calculated by coding each negative response with " 1 ", and otherwise " 0 "; the composite score (obtained by summing the individual preventive measure's score for each participant) ranged from 0 to 4 (Cronbach's alpha 0.7).

\section{Enabling/disabling factors}

Enabling factors included perceived socio-economic status, area of residence (rural/village, sub-urban setting or urban slum, and urban setting/city/town), being a student or worker in the health care sector, and most trusted source of COVID-19 information/advice (whereby the response categories 'other', including family and friends, 'radio/TV', 'social media', and 'religious authorities' were all coded as " 0 ", and 'health personnel' coded as 1). Perceived socio-economic status was sourced from the question, "Which of the following categories best describes your current socio-economic situation? Low-income category, lower-middle income category, upper-middle income category, and highincome category." Correct COVID-19 knowledge was defined as all three affirmative responses to "(1) if there is a possibility of being reinfected after recovering from a previous COVID-19 infection (Yes/No); (2) if COVID-19 infection could be prevented by a vaccine (Yes/No); and (3) if there is currently an effective vaccine against COVID-19 (Yes/No)." [22].

Disabling factors included the assessment of psychological distress with the Patient Health Questionnaire (PHQ-4) for Depression and Anxiety symptoms $[23,24]$. The PHQ-4 is a valid and reliable measure of depression and anxiety symptoms in the general population. PHQ-4 is a 4-item inventory rated on a four-point Likert scale. The severity of psychological distress was categorized as "normal (0-2), mild (3-5), 
moderate (6-8) and severe (9-12) based on the PHQ-4 scores" [23].

Need for care factors included three questions on 1) the level of fear/worry of being infected with COVID19 (ranging from $1=$ not at all worried to $5=$ extremely worried), 2) having been quarantined (either at home or elsewhere) at any point in time during the COVID-19 epidemic, and 3) chronic/underlying diseases including heart disease, hypertension, diabetes, cancer, HIV, tuberculosis, and chronic asthma; coded as "0" none and " 1 " at least presence of one clinically diagnosed condition.

\section{Data analysis}

Survey weights were used to control the proportion contribution of each participating country to the total population (aged 15 and above in each country in 2019) estimate. The country-specific weight was used to adjust for any deviations in the estimates due to bias arising from over- and under-coverage. More details about the survey weights were provided previously [22].

Descriptive statistics were used to describe the study population. Logistic regression was used to assess associations between predisposing factors, enabling and disabling factors, need of care factors and COVID-19 testing status, COVID-19 positive versus negative status and COVID-19 positive versus negative and not tested status. Variables significant at $<0.05$ in univariate analyses were subsequently included in the multivariable logistic regression models. $P$-values at $<0.05$ were considered significant. Statistical analyses were conducted using "STATA software version 15.0" (Stata Corporation, College Station, Texas, USA).

\section{Results}

In total, 10,183 participants were included in the study (6470 Brazil, 1738 Malaysia, 1124 Thailand, 230 Bangladesh, 219 Democratic Republic of Congo (DRC), 159 Benin, 107 Uganda, 81 Malawi and 55 Mali), with median age 45 years, interquartile range 33-57 years, range 18-93 years. Of these, $40.3 \%$ knew their COVID-19 infection status during the ongoing pandemic: $7.3 \%$ had tested positive and $33.0 \%$ had tested negative.

\section{Predisposing factors}

One in five participants (20.2\%) were 60years and older, $64.9 \%$ were female, $90.8 \%$ lived with other people, $47.5 \%$ had a postgraduate education, and $47.8 \%$ did not adhere to the four COVID-19 preventive measures.

\section{Enabling/disabling factors}

Most participants (80.4\%) resided in urban areas, $48.0 \%$ rated their socio-economic status as upper middle to high-income, and $34.4 \%$ were students or workers in health care. The most trusted source of COVID-19 information/advice was health care personnel (61.7\%); $51.5 \%$ had correct COVID-19 knowledge, and 18.5\% reported moderate to severe psychological distress.

\section{Need for care factors}

More than half of the participants (51.7\%) were very or extremely worried about being (re)infected with COVID$19,38.2 \%$ had been quarantined during the COVID-19 pandemic, and $29.2 \%$ had at least one diagnosed chronic/ underlying disease (see Table 1).

\section{Associations with COVID-19 testing}

In the adjusted logistic regression model, predisposing factors (residing in Brazil, having attained postgraduate education), enabling/disabling factors (urban residence, higher perceived economic status, being a student or worker in the health care sector, and reporting moderate or severe psychological distress), and need factors (having at least one chronic condition) increased the odds of COVID-19 testing. In addition, in the unadjusted analysis, correct COVID-19 knowledge and worry or fear of being (re)infected with COVID-19 were positively associated with COVID-19 testing status (Table 2).

Regarding the odds of COVID-19 positive diagnosis among all participants who reported COVID-19 test results in the adjusted analysis, residing in Bangladesh, and moderate to severe psychological distress were positively associated with COVID-19 positive diagnosis. Meanwhile, residing in Malaysia or Thailand and a higher education level were negatively associated with a COVID-19 positive diagnosis. In addition, in the unadjusted analysis, older age, urban residence, and higher perceived economic status were also associated with COVID-19 positivity (Table 2).

Adjusted logistic regression of the odds of a COVID19 positive diagnosis among all individuals tested or not tested found that urban residence, higher perceived economic status, being a student or worker in the health sector, and moderate or severe psychological distress were positively associated with a COVID-19 positive diagnosis, and residing in Malaysia, Thailand or five African countries was negatively associated with a COVID-19 positive diagnosis. In addition, in the unadjusted analysis, older age was negatively, and female sex was positively associated with a COVID-19 positive diagnosis (see Table 2).

\section{Discussion}

This study conducted among adults in nine LMIC from December 2020 to February 2021 showed that the COVID-19 testing uptake was $40.3 \%$ and that $7.3 \%$ tested SARS-CoV-2 positive, which is higher than in a 
Table 1 Sample and COVID-19 testing characteristics of adults in nine low-and middle-income countries, 2020, 2021

\begin{tabular}{|c|c|c|c|c|}
\hline \multirow[t]{3}{*}{ Variable } & \multirow{3}{*}{$\begin{array}{l}\text { Sample } \\
\mathrm{N}(\%)\end{array}$} & \multicolumn{3}{|c|}{ COVID-19 testing/infection status } \\
\hline & & \multirow{2}{*}{$\begin{array}{l}\text { Not tested/no results } \\
\mathrm{N}(\%)\end{array}$} & \multirow{2}{*}{$\begin{array}{l}\text { Negative } \\
N(\%)\end{array}$} & \multirow{2}{*}{$\begin{array}{l}\text { Positive } \\
\text { N (\%) }\end{array}$} \\
\hline & & & & \\
\hline All & 10,183 & $6078(59.7)$ & $3362(33.0)$ & $743(7.3)$ \\
\hline \multicolumn{5}{|l|}{ Predisposing factors } \\
\hline \multicolumn{5}{|l|}{ Country } \\
\hline Brazil & $6470(63.5)$ & $3283(50.7)$ & $2526(39.0)$ & $61(10.2)$ \\
\hline Malaysia & $1738(17.1)$ & $1246(71.7)$ & $473(27.2)$ & $19(1.1)$ \\
\hline Thailand & $1124(11.0)$ & $1017(90.5)$ & $104(9.3)$ & $3(0.3)$ \\
\hline Bangladesh & $230(2.3)$ & $133(57.8)$ & $60(26.1)$ & $37(16.1)$ \\
\hline 5 African countries & $621(6.1)$ & $399(64.3)$ & $199(32.0)$ & $23(3.7)^{\mathrm{a}}$ \\
\hline \multicolumn{5}{|l|}{ Age in years } \\
\hline $18-39$ & $4041(39.7)$ & $2397(59.3)$ & $1343(33.2)$ & $301(7.4)$ \\
\hline $40-59$ & $4081(40.1)$ & $2367(58.0)$ & $1408(34.5)$ & $306(7.5)$ \\
\hline 60 or more & $2061(20.2)$ & $1314(63.8)$ & $611(29.6)$ & $136(6.6)$ \\
\hline \multicolumn{5}{|l|}{ Sex } \\
\hline Male & $3579(35.1)$ & $2164(60.5)$ & $1178(32.9)$ & $237(6.6)$ \\
\hline Female & $6604(64.9)$ & $3914(59.3)$ & $2184(33.1)$ & $506(7.7)$ \\
\hline \multicolumn{5}{|l|}{ Living status } \\
\hline Lives alone & $932(9.2)$ & $528(56.7)$ & $329(35.3)$ & $75(8.0)$ \\
\hline Lives with other people & $9251(90.8)$ & $5550(60.0)$ & $3033(32.8)$ & $668(7.2)$ \\
\hline \multicolumn{5}{|l|}{ Education } \\
\hline Primary/secondary & $1316(13.0)$ & $838(67.7)$ & $315(25.4)$ & $85(6.9)$ \\
\hline Undergraduate & $4028(39.5)$ & $2612(64.8)$ & $1163(28.9)$ & $253(6.3)$ \\
\hline Postgraduate & $4839(47.5)$ & $2567(53.0)$ & $1873(38.7)$ & $399(8.2)$ \\
\hline \multicolumn{5}{|l|}{ Preventive measures } \\
\hline Not social distancing & $3378(33.2)$ & $1910(56.5)$ & $1124(33.3)$ & $344(10.2)$ \\
\hline Not wearing face mask & $413(4.1)$ & $231(55.9)$ & $136(32.9)$ & $46(11.1)$ \\
\hline No hand hygiene & $1154(11.3)$ & $680(58.9)$ & $356(30.8)$ & $118(10.2)$ \\
\hline No coughing hygiene & $3372(33.1)$ & $1986(58.9)$ & $1112(33.0)$ & $274(8.1)$ \\
\hline Not all four measures & $4872(47.8)$ & $2879(47.4)$ & $1570(46.7)$ & $423(56.9)$ \\
\hline \multicolumn{5}{|l|}{ Enabling/disabling factors } \\
\hline \multicolumn{5}{|l|}{ Residence } \\
\hline Rural & $812(8.0)$ & $654(80.5)$ & $145(17.9)$ & $13(1.6)$ \\
\hline Suburban/urban slum & $1185(11.6)$ & $776(65.5)$ & $334(28.2)$ & $75(6.3)$ \\
\hline Urban & $8186(80.4)$ & $4648(56.8)$ & $2883(35.2)$ & $655(8.0)$ \\
\hline \multicolumn{5}{|l|}{ Income } \\
\hline Low/lower middle & $5298(52.0)$ & $3522(66.5)$ & $1450(27.4)$ & $326(6.2)$ \\
\hline Upper middle/high & $4885(48.0)$ & $2556(52.3)$ & $1912(39.1)$ & $417(8.5)$ \\
\hline Student/worker in healthcare & $3500(34.4)$ & $1780(50.9)$ & $1414(40.4)$ & $306(8.7)$ \\
\hline \multicolumn{5}{|c|}{ Most trusted source of COVID-19 information/advice } \\
\hline Other & $3905(38.3)$ & $2444(62.6)$ & $1217(31.2)$ & $244(6.2)$ \\
\hline Health care personnel & $6278(61.7)$ & $3634(57.9)$ & $2145(34.2)$ & $499(7.9)$ \\
\hline COVID-19 correct knowledge & $5145(51.5)$ & $2885(55.0)$ & $1932(36.8)$ & $428(8.2)$ \\
\hline \multicolumn{5}{|l|}{ Psychological distress } \\
\hline None & $4774(46.9)$ & $3057(64.0)$ & $1442(30.2)$ & $275(5.8)$ \\
\hline Mild & $3528(34.6)$ & $2010(57.0)$ & $1244(35.3)$ & $274(7.8)$ \\
\hline Moderate & $1152(11.3)$ & $618(53.6)$ & $418(36.3)$ & $116(10.1)$ \\
\hline Severe & $729(7.2)$ & 393 (53.9) & $258(35.4)$ & 78 (10.7) \\
\hline
\end{tabular}


Table 1 (continued)

\begin{tabular}{|c|c|c|c|c|}
\hline \multirow[t]{3}{*}{ Variable } & \multirow{3}{*}{$\begin{array}{l}\text { Sample } \\
\mathrm{N}(\%)\end{array}$} & \multicolumn{3}{|c|}{ COVID-19 testing/infection status } \\
\hline & & \multirow{2}{*}{$\begin{array}{l}\text { Not tested/no results } \\
\mathrm{N}(\%)\end{array}$} & \multirow{2}{*}{$\begin{array}{l}\text { Negative } \\
\mathrm{N}(\%)\end{array}$} & \multirow{2}{*}{$\begin{array}{l}\text { Positive } \\
\text { N (\%) }\end{array}$} \\
\hline & & & & \\
\hline \multicolumn{5}{|l|}{ Need for care factors } \\
\hline $\begin{array}{l}\text { Worry/fear about being (re) infected with COVID-19 (very } \\
\text { or extremely) }\end{array}$ & $5160(51.7)$ & $3074(58.4)$ & $1818(34.6)$ & $368(7.0)$ \\
\hline Has been quarantined during COVID-19 epidemic & $3889(38.2)$ & $1576(40.4)$ & $1656(42.6)$ & $657(16.9)$ \\
\hline Chronic condition(s) total & $2974(29.2)$ & $1683(56.6)$ & $1072(36.0)$ & $219(7.4)$ \\
\hline Heart disease & $419(4.1)$ & $248(59.2)$ & $144(34.4)$ & $27(6.4)$ \\
\hline Hypertension & $1825(17.9)$ & $1053(57.7)$ & $648(35.6)$ & $123(6.7)$ \\
\hline Diabetes & $694(6.8)$ & $425(61.2)$ & $219(31.6)$ & $50(7.2)$ \\
\hline Cancer & $157(1.5)$ & $80(51.0)$ & $64(40.8)$ & $13(8.3)$ \\
\hline HIV & $56(0.5)$ & $26(46.4)$ & $25(44.6)$ & $5(8.9)$ \\
\hline Tuberculosis & $14(0.1)$ & $6(42.9)$ & $5(35.7)$ & $3(21.4)$ \\
\hline Asthma & $730(7.2)$ & $375(51.4)$ & $295(40.4)$ & $60(8.2)$ \\
\hline
\end{tabular}

Ranging from 2.5\% in Benin and Malawi to 7.3\% in Mali

large community survey in Canada (3.3\% of those who had undergone testing were tested positive) [2] and in Germany ( 0.46 to $1.82 \%$ of the population-based cohort were tested positive) [5]. The high proportion of COVID19 testing uptake and the higher proportion of positive SARS-CoV-2 tests may be partially attributed to the high participation rate of students or workers in the health care sector $(34.4 \%)$ but also because in LMIC testing is more likely to be done among symptomatic individuals or persons in contact with positive cases.

Using the Andersen's health care utilization model, we found that predisposing factors (residing in Brazil, postgraduate education), enabling/disabling factors (urban residence, higher perceived economic status, being a student or worker in the health care sector, and moderate or severe psychological distress), and need factors (having at least one chronic condition) increased the odds of COVID-19 testing. Previous studies also found that several underlying health conditions, higher household income, and occupation (health care worker) were associated with COVID-19 testing [2, 3]. Persons with underlying health conditions such as obesities, diabetes and cardiovascular diseases are at increased risk for developing severe diseases and therefore tested when they develop COVID-19 like symptoms [25]. People with higher education and higher economic status may have better access to health information, such as the importance of knowing one's COVID-19 status. Unlike previous studies that found an association between older age, male sex, and uptake of COVID-19 testing [2, 3], we did not find age and sex differences in the uptake of COVID19 testing. It is interesting to note the demographic differences in COVID-19 testing in LMICs, compared with previous studies conducted in Canada and the UK $[2,3]$. Perhaps there was no concerted effort to test the elderly among the LMIC countries, unlike in Canada where long-term care facilities contributed significantly to COVID-19 mortality, necessitating the mass testing of elderly individuals [26]. Compared to Brazil, all other study countries had a lower uptake of COVID-19 testing. High COVID-19 testing was also observed among health care workers in Brazil (73.6\%) [27]. This high uptake of testing in Brazil is explained by the high COVID-19 disease burden in Brazil and the widespread use of COVID19 rapid antigen tests $[28,29]$.

Regarding the odds of COVID-19 positive diagnosis among all persons tested, residing in Bangladesh, and moderate to severe psychological distress were positively associated with COVID-19 positive diagnosis and residing in Malaysia and Thailand and higher education were negatively associated with a COVID-19 positive diagnosis. In the UK biobank data study, ethnicity (non-Whites) and lower education were associated with COVID-19 positive diagnosis [3]. However, both in this study and the UK biobank data study among tested persons, no significant association between comorbidities or health risk factors with a COVID-19 positive diagnosis was found [3].

The association between psychological distress and COVID-19 positive diagnosis has also been found among COVID-19 survivors in China [30] and may be attributed to stress reactions following the COVID-19 positive diagnosis potentially leading to long-term mental disorders [30]. In another study in China, depressive symptoms were more prevalent in COVID-19 patients compared to non-COVID-19 controls and were associated with an 
Table 2 Associations with COVID-19 testing and COVID-19 diagnosis

\begin{tabular}{|c|c|c|c|c|c|c|}
\hline \multirow[t]{2}{*}{ Variable } & \multicolumn{2}{|c|}{ Odds of COVID-19 testing $(95 \% \mathrm{Cl})$} & \multicolumn{2}{|c|}{$\begin{array}{l}\text { Odds of COVID-19 diagnosis, among } \\
\text { all individuals tested }(95 \% \mathrm{Cl})\end{array}$} & \multicolumn{2}{|c|}{$\begin{array}{l}\text { Odds of COVID-19 diagnosis, among all } \\
\text { individuals tested or not tested ( } 95 \% \mathrm{CI}\end{array}$} \\
\hline & Unadjusted & Adjusted & Unadjusted & Adjusted & Unadjusted & Adjusted \\
\hline \multicolumn{7}{|l|}{ Predisposing factors } \\
\hline \multicolumn{7}{|l|}{ Country } \\
\hline Brazil & 1 (Reference) & 1 (Reference) & 1 (Reference) & 1 (Reference) & 1 (Reference) & 1 (Reference) \\
\hline Malaysia & $0.41(0.36,0.46)^{* * *}$ & $0.51(0.44,0.59)^{* * *}$ & $0.15(0.10,0.24)^{* * *}$ & $0.15(0.09,0.24)^{* * *}$ & $0.10(0.06,0.15)^{* * *}$ & $0.13(0.08,0.20)^{* * *}$ \\
\hline Thailand & $0.11(0.09,13.3)^{* * *}$ & $0.14(0.11,0.18)^{* * *}$ & $0.11(0.03,0.35)^{* * *}$ & $0.14(0.04,0.44)^{* * *}$ & $0.02(0.01,0.07)^{* * *}$ & $0.04(0.01,0.11)^{* * *}$ \\
\hline Bangladesh & $0.75(0.58,0.98)^{*}$ & $0.62(0.46,0.83)^{* *}$ & $2.36(1.55,3.58)^{* * *}$ & $1.98(1.24,3.16)^{* *}$ & $1.68(1.17,2.42)^{* *}$ & $1.41(0.94,2.13)$ \\
\hline 5 African countries & $0.57(0.48,0.68)^{* * *}$ & $0.53(0.53,0.88)^{* *}$ & $0.44(0.28,0.68)^{* * *}$ & $0.52(0.31,0.86)$ & $0.34(0.22,0.52)^{* * *}$ & $0.37(0.22,0.64)^{* * *}$ \\
\hline \multicolumn{7}{|l|}{ Age in years } \\
\hline $18-39$ & 1 (Reference) & - & 1 (Reference) & 1 (Reference) & 1 (Reference) & 1 (Reference) \\
\hline $40-59$ & $1.09(0.93,1.27)$ & & $0.60(0.43,0.83)^{* *}$ & $0.98(0.68,1.40)$ & $0.68(0.50,0.93)^{*}$ & $1.16(0.81,1.64)$ \\
\hline 60 or more & $1.04(0.87,1.23)$ & & $0.61(0.44,0.86)^{* *}$ & $0.89(0.60,1.34)$ & $0.67(0.50,0.92)^{*}$ & $0.85(0.60,1.21)$ \\
\hline \multicolumn{7}{|l|}{ Sex } \\
\hline Male & 1 (Reference) & - & 1 (Reference) & - & 1 (Reference) & 1 (Reference) \\
\hline Female & $1.07(0.91,1.26)$ & & $1.43(0.99,2.06)$ & & $1.42(1.00,2.00)^{*}$ & $1.06(0.73,1.53)$ \\
\hline \multicolumn{7}{|l|}{ Living status } \\
\hline Lives alone & 1 (Reference) & - & 1 (Reference) & - & 1 (Reference) & - \\
\hline Lives with other people & $1.00(0.82,1.22)$ & & $1.32(0.92,1.90)$ & & $1.27(0.90,1.79)$ & \\
\hline \multicolumn{7}{|l|}{ Education } \\
\hline Primary/secondary & 1 (Reference) & 1 (Reference) & 1 (Reference) & 1 (Reference) & 1 (Reference) & - \\
\hline Undergraduate & $1.22(0.92,1.61)$ & $1.17(0.87,1.56)$ & $0.57(0.31,1.03)$ & $0.53(0.30,0.94)^{*}$ & $0.74(0.42,1.31)$ & \\
\hline Postgraduate & $2.01(1.53,2.64)^{* * *}$ & $1.48(1.10,1.99)^{* *}$ & $0.43(0.24,0.75)^{* *}$ & $0.41(0.27,0.72)^{* *}$ & $0.81(0.47,1.40)$ & \\
\hline $\begin{array}{l}\text { Not adhering to } 4 \text { COVID-19 } \\
\text { preventive measures }\end{array}$ & $0.88(0.75,1.06)$ & - & $1.24(0.87,1.76)$ & - & $1.27(0.90,1.79)$ & - \\
\hline \multicolumn{7}{|l|}{ Enabling/disabling factors } \\
\hline \multicolumn{7}{|l|}{ Residence } \\
\hline Rural & 1 (Reference) & 1 (Reference) & 1 (Reference) & 1 (Reference) & 1 (Reference) & 1 (Reference) \\
\hline Suburban/urban slum & $2.74(1.95,3.84)^{* * *}$ & $1.25(0.86,1.84)$ & $3.83(1.65,8.87)^{* *}$ & $1.65(0.72,3.83)$ & $7.50(3.38,16.66)^{* * *}$ & $2.28(0.98,5.35)$ \\
\hline Urban & $4.66(3.61,6.01)^{* * *}$ & $1.89(1.40,2.57)^{* * *}$ & $3.85(1.99,7.44)^{* * *}$ & $1.60(0.82,3.09)$ & $10.90(5.84,20.36)^{* * *}$ & $3.11(1.57,6.15)^{* * *}$ \\
\hline \multicolumn{7}{|l|}{ Income } \\
\hline Low/lower middle & 1 (Reference) & 1 (Reference) & 1 (Reference) & 1 (Reference) & 1 (Reference) & 1 (Reference) \\
\hline Upper middle/high & $2.14(1.82,2.52)^{* * *}$ & $1.49(1.24,1.79)^{* * *}$ & $1.59(1.14,2.23)^{* *}$ & $1.20(0.85,1.70)$ & $2.47(1.80,3.41)^{* * *}$ & $1.59(1.15,2.19)^{* *}$ \\
\hline $\begin{array}{l}\text { Student or worker in the } \\
\text { healthcare sector }\end{array}$ & $1.57(1.34,1.85)^{* * *}$ & $2.05(1.70,2.48)^{* * *}$ & $1.37(0.98,1.91)$ & - & $1.77(1.29,2.42)^{* * *}$ & $1.93(1.37,2.72)^{* * *}$ \\
\hline \multicolumn{7}{|c|}{ Most trusted source of COVID-19 information/advice } \\
\hline Other & 1 (Reference) & - & 1 (Reference) & - & 1 (Reference) & - \\
\hline Health care personnel & $1.07(0.91,1.27)$ & & $1.32(0.91,1.91)$ & & $1.33(0.93,1.89)$ & \\
\hline $\begin{array}{l}\text { COVID-19 correct knowl- } \\
\text { edge }\end{array}$ & $1.36(1.15,1.58)^{* * *}$ & $0.95(0.78,1.16)$ & $0.86(0.61,1.20)$ & - & $1 / 07(0.78,1.48)$ & - \\
\hline \multicolumn{7}{|l|}{ Psychological distress } \\
\hline None & 1 (Reference) & 1 (Reference) & 1 (Reference) & 1 (Reference) & 1 (Reference) & 1 (Reference) \\
\hline Mild & $1.26(1.06,1.50)^{* *}$ & $1.05(0.85,1.29)$ & $1.17(0.80,1.73)$ & $1.11(0.73,1.71)$ & $1.35(0.93,1.95)$ & $1.07(0.71,1.59)$ \\
\hline Moderate & $1.82(1.37,2.42)^{* * *}$ & $1.40(1.03,1.91)^{*}$ & $2.57(1.51,4.36)^{* * *}$ & $1.98(1.16,3.39)^{*}$ & $3.24(1.97,5.33)^{* * *}$ & $1.22(1.32,3.74)^{* *}$ \\
\hline Severe & $1.63(1.15,2.32)^{* *}$ & $1.40(1.00,1.98)^{*}$ & $3.21(1.75,5.88)^{* * *}$ & $2.33(1.28,4.25)^{* *}$ & $3.58(2.02,6.34)^{* * *}$ & $2.58(1.45,4.60)^{* * *}$ \\
\hline \multicolumn{7}{|l|}{ Need for care factors } \\
\hline $\begin{array}{l}\text { Worry/fear about being (re) } \\
\text { infected with COVID-19 }\end{array}$ & $1.08(1.02,1.15)^{*}$ & $0.97(0.89,1.05)$ & $1.03(0.90,1.17)$ & - & $1.08(0.96,1.22)$ & - \\
\hline Chronic condition(s) & $1.44(1.21,1.72)^{* * *}$ & $1.25(1.02,1.52)^{*}$ & $0.95(0.64,1.39)$ & - & $1.21(0.84,1.75)$ & - \\
\hline
\end{tabular}

Cl Confidence Interval; *** $p<0.001 ;{ }^{* *} p<0.01 ;{ }^{*} p<0.05$ 
increase in inflammation markers [31]. Consequently, COVID-19 survivors should be screened for stress disorder, anxiety, and depression regularly to identify those with psychological distress for timely intervention [30]. The lower odds of COVID-19 positive diagnosis, among those with higher education can be explained by the significantly higher rate of COVID-19 testing among those with higher compared to those with lower education. Furthermore, it is possible that people with higher education and more access to quality information are more likely to take up vaccination, follow COVID-19 prevention measures and hence are less likely to become infected [32, 33]. The higher rate of COVID-19 positive diagnosis in Bangladesh may be explained by a higher prevalence of COVID-19 pandemic in Bangladesh (15.1\%) [12], and the reluctance of people to get tested for COVID-19, when they have no or only moderate COVID-19 symptoms [34]. The lower rate of COVID-19 positive diagnosis in Malaysia may be attributed to a lower average test positive ratio and testing rate (4.3\% and 0.8 tests per 1000 population) [11]. In Thailand the low rate of COVID-19 positive diagnosis may be attributed to a low prevalence of COVID-19 infection (7.5\% among hospital patients) [13], and a lowtest positive ratio (1.3, January-March 2021) [35], and a high compliance to COVID-19 preventive measures in Thailand [36].

The odds of a COVID-19 positive diagnosis among all individuals tested or not tested found increased with urban residence, higher perceived economic status, being a student or worker in the health sector, moderate or severe psychological symptoms, and not residing in Malaysia, Thailand or five African countries. We did not find a significant association between older age and having comorbidities and a COVID-19 positive diagnosis, as found in the Canadian study [2]. Other studies have likewise found that those who reside in urban areas are more likely to contract the COVID-19 virus compared to rural areas and small towns $[37,38]$. This has been attributed to population density and the occurrence of large gatherings more common in urban areas, which accelerated the disease transmission through respiratory droplets in crowded conditions [39]. The higher odds of those who have higher perceived economic status being COVID-19 positive may be attributed to their higher likelihood of being tested, as was found in this study and in another study in New York City [40]. These results may point to the disparity in healthcare access, in which individuals from a higher socioeconomic status have better chances of being tested positive and therefore able to seek treatment. Finally, it is not surprising that workers and students in the healthcare sectors had higher odds of being tested COVID-19 positive due to their higher exposure to the COVID-19 virus.
Like the UK study [3], we found that having comorbidities increased the odds of COVID-19 testing but not with the risk of testing positive. This finding may suggest that having comorbidities may assist in predicting the risk of developing COVID-19 symptoms, and therefore the probability of getting a COVID-19 test [3]. In a study in Bangladesh, underlying health conditions/non-communicable diseases triggered factors for anxiety and depression symptoms. This worry/ fear might be responsible for a higher uptake in testing [41].

Higher education was found associated with COVIDtesting uptake, but within the tested population lower education predicted a COVID-19 positive diagnosis. Individuals with lower education may be involved in manual or outdoor jobs which expose them to crowded working conditions (such as in factories), compared to those who have a higher education who may have the opportunity to work from their homes, and have more possibilities to adapt their working conditions to the pandemic situation [42, 43]. In addition, adherence to preventive measures has also been found to be lower among individuals with lower educational attainment [43]. Comparing COVID-19 positive diagnosis among those who tested and the whole sample, including those that had not tested, we found similarities on country, age, sex, living status, chronic conditions, not adhering to four COVID19 preventive measures, correct COVID-19 knowledge, psychological distress and worry/fear about being (re) infected with COVID-19, but we also found differences in terms of higher education negatively associated with COVID-19 positive diagnosis within the tested population, and an association between urban residence, higher perceived economic status, being a student or worker in the health care sector and COVID-19 positive diagnosis in the whole study population. Health care workers have been identified as a high-risk group for contracting and spreading the COVID-19 virus, due to exposure to symptomatic and asymptomatic patients in healthcare settings, a lack of adherence to preventive measures, and the lack of personal protective equipment [4].

Although a high proportion (51.7\%) of participants were very or extremely worried about being (re)infected with COVID-19, this translated only in the unadjusted analysis to higher odds of COVID-19 testing uptake, while higher psychological distress was associated with higher COVID-19 testing, and more severe psychological distress with a COVID-19 positive diagnosis. Individuals who have contracted the virus were found to exhibit more depression and anxiety symptoms in other studies. In a study in Italy among adults surviving COVID-19 infection, $31 \%$ presented depression symptoms and $42 \%$ anxiety symptoms [44]. It is possible that the psychological distress increased after COVID-19 positive diagnosis, 
but since survey was only a cross-sectional study, we are not able to determine the direction of the relationship between psychological distress, testing and testing positive. However, within the tested population, psychological distress was inversely associated with a COVID-19 negative diagnosis (analysis not shown).

\section{Study limitations}

Our study respondents cannot be considered representative of the general population in the study countries since respondents needed to have had access to the internet to participate in the online survey. We acknowledged this limitation of this study and do believe that studies like this one help in health surveillance actions, which directly impact the evolution of the pandemic in a country and the selection of more assertive preventive measures. Moreover, self-reports, including the outcome variable COVID-19 testing, may be influenced by recall bias and social desirability. Another potential limitation was the poor quality of certain data, e.g., the informational quality of information provided by health care personnel and other information sources. In addition, cultural differences between countries and between regions of the same country can be a limitation in relation to the quality of the information. We also have no information on the type of test used for COVID-19 testing, limiting the accuracy and authenticity of the test results. Some variables, such as COVID-19 symptoms, previous use of health care services, obesity, and smoking, that have been found affecting COVID-19 testing uptake $[2,3]$ were not assessed in this survey and should be included in future studies.

\section{Conclusions}

This study among adults across nine LMICs reported a high prevalence of COVID-19 testing. Factors associated with COVID-19 testing included predisposing factors (residing in Brazil, postgraduate education), enabling/ disabling factors (urban residence, higher perceived economic status, being a student or worker in the health care sector, and moderate or severe psychological distress), and need factors (having at least one chronic condition). Identified predisposing and enabling or disabling factors can be used to design programmes to improve COVID19 testing uptake. Access to testing needs to be increased for persons living in Africa and similar resource poor settings. In addition, COVID-19 testing programs need to target persons of lower economic status and of lower education level who currently less tested but most at risk for COVID-19 infection.
Abbreviations

LMCl: Low- and middle-income countries; PHQ: Patient Health Questionnaire; PM: particulate matter.

\section{Acknowledgements}

We would like to thank all our participants.

All methods were carried out in accordance with relevant guidelines and regulations.

\section{Authors' contributions}

All authors fulfil the criteria for authorship. Conceptualization, SP, KP, SAB, EFdMV, JNSF, and RC; methodology, SP, KP, SAB, EFdMV, CSS, WSC, MTH, PS, JDD, IJC, MCH, HD, JNSF, and RC; software, WSC; formal analysis, SP, KP, WSC, and JNSF; data curation. SAB, CSS, and WSC; writing - original draft preparation, SP, KP, SAB, CSS, WSC, and RKW; writing-all authors reviewed and edited the manuscript; supervision, SP, KP, SAB, EFdMV, and RC; project administration, SAB, EFdMV, SP, and RC.; funding acquisition, RC, SP. All authors read and approved the final version of the manuscript and have agreed to the authorship and order of authorship for this manuscript.

\section{Funding}

This research was funded by VLIRUOS (Flemish Interuniversity Council for University Development Cooperation) and Mahidol University.

\section{Availability of data and materials}

Data are available upon reasonable request. Data are available on the International Consortium (International Citizen Project COVID-19 (ICPcovid): http:// www.icpcovid. com) website and could be used by other investigators on request. De-identified participant data are available from Joseph Nelson Siewe Fodjo at Email: josephnelson.siewefodjo@uantwerpen.be

\section{Declarations}

\section{Ethics approval and consent to participate}

The study was conducted according to the guidelines of the Declaration of Helsinki, and were approved by the institutional review boards in the following countries: Brazil: National Research Ethics Commission, CAAE: 30343820.9.0000.0008, report number 4.518.164; Malaysia: University Malaya Research Ethics Committee, protocol code UM.TNC2/UMREC_1165, 27 January 2021; Thailand: The Committee for Research Ethics (Social Sciences), Mahidol University, ethics code 2020/127 (B1); Bangladesh: Biosafety, Biosecurity \& Ethical Committee, Faculty of Biological Sciences, Jahangirnagar University, Savar, Dhaka, Bangladesh, ethics code: BBEC, JU/ M-2020 (6)I/ RG/ Bangladesh; DRC: National Ethical Committee, reference number CNES N_175/CNES/BN/PMMF/2020; Benin: National Ethics Committee for Health Research (CNERS) of Benin, ethical opinion N_21 of 7 May 2020; Uganda: The Ethics committee of School of Public health, Makerere University, Kampala, Uganda, HDREC number 809; Malawi: University of North Carolina Institutional Review Board and the Malawi National Health Sciences Research Committee reviewed the protocol and provided an exemption as "human subjects research" given the online survey nature of the work; Mali: Ethical Committee of the Faculty of Medicine and OdontoStomatology / Faculty of Pharmacy of Bamako, approval number (2020/56/CE/FMOS/FAPH); Belgium: The Ethics Committee of the University Hospital Antwerp 20/13/148. Informed consent was obtained from all participants.

\section{Consent for publication}

Not applicable.

\section{Competing interests}

The authors declare that they have no competing interests.

\section{Author details}

${ }^{1}$ Department of Health Education and Behavioral Sciences, Faculty of Public Health, Mahidol University, Bangkok 10400, Thailand. ${ }^{2}$ Department of Research Administration and Development, University of Limpopo, Polokwane, South Africa. ${ }^{3}$ Department of Psychology, College of Medical and Health Science, Asia University, Taichung, Taiwan. ${ }^{4}$ Disease Control Coordination, São Paulo State Health Department, São Paulo 01246-000, Brazil. ${ }^{5}$ Institute of Tropical 
Pathology and Public Health, Federal University of Goiás, Goiânia 74690-900, Brazil. ${ }^{6}$ Global Health Institute, University of Antwerp, 2000 Antwerp, Belgium. ${ }^{7}$ Centre for Community Health Studies (ReaCH), Faculty of Health Sciences, Universiti Kebangsaan Malaysia, 50300 Kuala Lumpur, Malaysia. ${ }^{8}$ Department of Health Science and Biostatistics, Faculty of Health, Arts and Design, Swinburne University of Technology, Hawthorn, VIC 3122, Australia. ${ }^{9}$ School of Social Science, Universiti Sains Malaysia, 11800 Gelugor, Malaysia. ${ }^{10}$ Institute of Nutrition, Mahidol University, Nakhon Pathom 73170, Thailand. ${ }^{11}$ Jeeon Bangladesh Ltd., Dhaka, Bangladesh. ${ }^{12}$ Department of Public Health, State University of Bangladesh (SUB), Dhaka, Bangladesh. ${ }^{13}$ Department of Primary Care \& Mental Health, University of Liverpool, Liverpool L69 3BX, UK. ${ }^{14}$ School of Public Health, College of Health Sciences, Makerere University, Kampala, Uganda. ${ }^{15}$ University of North Carolina UNC Project Malawi, Lilongwe, Malawi. ${ }^{16}$ International Center of Excellence in Research, Faculty of Medicine and OdontoStomatology, Bamako, Mali. ${ }^{17}$ Lymphatic Filariasis Research Unit/ International Center of Excellence in Research, Bamako, Mali. ${ }^{18}$ Research Unit on Communicable Diseases, Polytechnic School of Abomey-Calavi, University of Abomey-Calavi, Cotonou 01, BP 526, Benin. ${ }^{19}$ Kinshasa School of Public Health, University of Kinshasa, Kinshasa 7948, Democratic Republic of the Congo.

Received: 8 July 2021 Accepted: 21 February 2022

Published online: 28 February 2022

\section{References}

1. National Institute of Aging. Why COVID-19 testing is the key to getting back to normal, 2020. URL: https://www.nia.nih.gov/news/why-covid-19testing-key-getting-back-normal (Accessed 5 July 2021).

2. Sundaram ME, Calzavara A, Mishra S, Kustra R, Chan AK, Hamilton $M A$, et al. Individual and social determinants of SARS-CoV-2 testing and positivity in Ontario, Canada: a population-wide study. CMAJ. 2021;193(20):E723-34. https://doi.org/10.1503/cmaj.202608.

3. Chadeau-Hyam M, Bodinier B, Elliott J, Whitaker MD, Tzoulaki I, Vermeulen $\mathrm{R}$, et al. Risk factors for positive and negative COVID-19 tests: a cautious and in-depth analysis of UK biobank data. Int J Epidemiol. 2020;49(5):1454-67. https://doi.org/10.1093/ije/dyaa134.

4. Gómez-Ochoa SA, Franco OH, Rojas LZ, Raguindin PF, Roa-Díaz ZM, Wyssmann BM, et al. COVID-19 in Health-Care Workers: A Living Systematic Review and Meta-Analysis of Prevalence, Risk Factors, Clinical Characteristics, and Outcomes. Am J Epidemiol. 2021;190(1):161-75. https://doi.org/ 10.1093/aje/kwaa191.

5. Pritsch M, Radon K, Bakuli A, Le Gleut R, Olbrich L, Noller G, et al. Prevalence and risk factors of infection in the representative COVID-19 cohort Munich. Int J Environ Res Public Health. 2021;18(7):3572.

6. Torres TS, Luz PM, Coelho LE, Jalil C, Falco GG, Sousa LP, et al. SARS-CoV-2 testing disparities across geographical regions from a large metropolitan area in Brazil: Results from a web-based survey among individuals interested in clinical trials for COVID-19 vaccines. Braz J Infect Dis. 2021;25(4):101600. https://doi.org/10.1016/j.bjid.2021.101600.

7. Andersen RM. Revisiting the behavioral model and access to medical care: does it matter? J Health Soc Behav. 1995;36(1):1-10.

8. Seidu AA. Using Anderson's Model of Health Service Utilization to Assess the Use of HIV Testing Services by Sexually Active Men in Ghana. Front Public Health. 2020;8:512. https://doi.org/10.3389/fpubh.2020.00512.

9. Li YN, Nong DX, Wei B, Feng QM, Luo HY. The impact of predisposing, enabling, and need factors in utilization of health services among rural residents in Guangxi, China. BMC Health Serv Res. 2016;16(1):592. https:// doi.org/10.1186/s12913-016-1825-4.

10. Hallal PC, Hartwig FP, Horta BL, Silveira MF, Struchiner CJ, Vidaletti LP, et al. SARS-CoV-2 antibody prevalence in Brazil: results from two successive nationwide serological household surveys. Lancet Glob Health. 2020;8(11):e1390-8. https://doi.org/10.1016/S2214-109X(20)30387-9.

11. Jayaraj VJ, Rampal S, Ng CW, Chong DWQ. The Epidemiology of COVID-19 in Malaysia. Lancet Reg Health West Pac. 2021;17:100295. https://doi.org/ 10.1016/j.lanwpc.2021.100295.

12. Rahman A, Marjan N, Afroz N, Afroz N, Hossain Z. Prevalence and transmission of COVID-19 in community and household levels of Bangladesh: Longini and Koopman epidemic modelling approach. Int J Clin Pract. 2021:e14921. https://doi.org/10.1111/ijcp.14921.
13. Sirijatuphat R, Suputtamongkol Y, Angkasekwinai N, Horthongkham N, Chayakulkeeree M, Rattanaumpawan P, et al. Epidemiology, clinical characteristics, and treatment outcomes of patients with COVID-19 at Thailand's university-based referral hospital. BMC Infect Dis. 2021;21(1):382. https://doi.org/10.1186/s12879-021-06081-z.

14. Nkuba AN, Makiala SM, Guichet E, Tshiminyi PM, Bazitama YM, Yambayamba MK, et al. High prevalence of anti-SARS-CoV-2 antibodies after the first wave of COVID-19 in Kinshasa, Democratic Republic of the Congo: results of a cross-sectional household-based survey. Clin Infect Dis. 2021:ciab515. https://doi.org/10.1093/cid/ciab515.

15. Our World in Data=a Total COVID-19 tests per 1,000 people. URL: https:// ourworldindata.org/grapher/full-list-cumulative-total-tests-per-thousand (Accessed 15 Jan 2022).

16. Our World in Data=b Total COVID-19 tests for each confirmed case. URL: https://ourworldindata.org/grapher/number-of-covid-19-tests-per-confi rmed-case-bar-chart (Accessed 15 Jan 2022).

17. Our World in Data=c COVID-19 vaccine doses administered per 100 people. URL: https://ourworldindata.org/grapher/covid-vaccinationdoses-per-capita (Accessed 15 Jan 2022).

18. Alcántara R, Peñaranda K, Mendoza-Rojas G, Nakamoto JA, Martins-Luna J, Del Valle-Mendoza J, et al. Unlocking SARS-CoV-2 detection in low- and middle-income countries. Cell Rep. Methods. 2021;100093. https://doi. org/10.1016/j.crmeth.2021.100093.

19. Connor A, Hariharan N, Carson S, Sanders KC, Vosburg KB, Sabot O. Access To COVID-19 Testing In Low- And Middle-Income Countries Is Still Critical To Achieving Health Equity. 2021. https://doi.org/10.1377/FOREFRONT. 20211026.483412. https://www.healthaffairs.org/do/10.1377/forefront. 20211026.483412/full/

20. International Citizens Project. International citizen project to assess adherence to public health measures and their impact on the COVID-19 outbreak, 2020. URL: https://www.icpcovid.com. 2020 (Accessed 10 Oct 2020).

21. Lieberoth A, Lin SY, Stöckli S, Han H, Kowal M, Gelpi R, et al. COVIDiSTRESS global survey consortium. Stress and worry in the 2020 coronavirus pandemic: relationships to trust and compliance with preventive measures across 48 countries in the COVIDiSTRESS global survey. R Soc Open Sci. 2021;8(2):200589. https://doi.org/10.1098/rsos.200589.

22. Bono SA, de Moura F, Villela E, Siau CS, Chen WS, Pengpid S, et al. Factors Affecting COVID-19 Vaccine Acceptance: An International Survey among Low- and Middle-Income Countries. Vaccines. 2021;9(5):515. https://doi. org/10.3390/vaccines9050515.

23. Kroenke K, Spitzer RL, Williams JB, Löwe B. An ultra-brief screening scale for anxiety and depression: the PHQ-4. Psychosomatics. 2009;50:613-21.

24. Löwe B, Wahl I, Rose M, Spitzer C, Glaesmer H, Wingenfeld K, et al. A 4-item measure of depression and anxiety: validation and standardization of the Patient Health Questionnaire-4 (PHQ-4) in the general population. J Affect Disord. 2010;122(1-2):86-95. https://doi.org/10. 1016/j.jad.2009.06.019.

25. Centers for Disease Control and Prevention (CDC). Science Brief: Evidence Used to Update the List of Underlying Medical Conditions Associated with Higher Risk for Severe COVID-19, 2021. URL: https://www.cdc.gov/ coronavirus/2019-ncov/science/science-briefs/underlying-evidencetable.html (Accessed 10 Oct 2021).

26. Liu M, Maxwell CJ, Armstrong P, Schwandt M, Moser A, McGregor MJ, et al. COVID-19 in long-term care homes in Ontario and British Columbia. CMAJ. 2020;192(47):E1540-6. https://doi.org/10.1503/cmaj.201860.

27. de Moura F, Villela E, Rodrigues da Cunha I, Nelson Siewe Fodjo J, Obimpeh M, Colebunders R, et al. Impact of COVID-19 on Healthcare Workers in Brazil between August and November 2020: A Cross-Sectional Survey. Int J Environ Res Public Health. 2021;18(12):6511. https://doi.org/ 10.3390/ijerph18126511.

28. Neiva MB, Carvalho I, Costa Filho EDS, Barbosa-Junior F, Bernardi FA, Sanches TLM, et al. Rev Soc Bras Med Trop. 2020;53:e20200550. https:// doi.org/10.1590/0037-8682-0550-2020 eCollection 2020.

29. Matsuda EM, de Campos IB, de Oliveira IP, Colpas DR, Carmo AMDS, Brígido LFM, et al. J Med Virol. 2021;93(7):4405-10. https://doi.org/10. 1002/jmv.26985.

30. Cai X, Hu X, Ekumi IO, Wang J, An Y, Li Z, et al. Psychological Distress and Its Correlates Among COVID-19 Survivors During Early Convalescence Across Age Groups. Am J Geriatr Psychiatry. 2020;28(10):1030-9. https:// doi.org/10.1016/j.jagp.2020.07.003. 
31. Guo Q, Zheng Y, Shi J, Wang J, Li G, Li C, et al. Immediate psychological distress in quarantined patients with COVID-19 and its association with peripheral inflammation: a mixed-method study. Brain Behav Immun. 2020;88:17-27.

32. Li PC, Theis SR, Kelly D, Ocampo T, Berglund A, Morgan D, et al. Impact of an Education Intervention on COVID-19 Vaccine Hesitancy in a Military Base Population. Mil Med. 2021:usab363. https://doi.org/10.1093/ milmed/usab363.

33. Humer E, Jesser A, Plener PL, Probst T, Pieh C. Education level and COVID19 vaccination willingness in adolescents. Eur Child Adolesc Psychiatry. 2021;1-3. https://doi.org/10.1007/s00787-021-01878-4.

34. Saha P, Gulshan J. Systematic Assessment of COVID-19 Pandemic in Bangladesh: Effectiveness of Preparedness in the First Wave. Front Public Health. 2021;9:628931. https://doi.org/10.3389/fpubh.2021.628931.

35. Rampal S, Rampal L, Jayaraj VJ, Pramanick A, Choolani M, Liew BS, et al. The epidemiology of COVID-19 in ten Southeast Asian countries. Med J Malaysia. 2021;76(6):783-91.

36. Triukose S, Nitinawarat S, Satian P, Somboonsavatdee A, Chotikarn P Thammasanya T, et al. Effects of public health interventions on the epidemiological spread during the first wave of the COVID-19 outbreak in Thailand. PLoS One. 2021;16(2):e0246274. https://doi.org/10.1371/journal. pone. 0246274

37. Paul R, Arif AA, Adeyemi O, Ghosh S, Han D. Progression of COVID-19 from urban to rural areas in the United States: a spatiotemporal analysis of prevalence rates. J Rural Health. 2020;36(4):591-601.

38. Kulu H, Dorey P. Infection rates from Covid-19 in Great Britain by geographical units: A model-based estimation from mortality data. Health Place. 2021;67:102460

39. Covid CD, Team R, COVID C, Team R, COVID C, Team R, Bialek S, Bowen V, Chow N, Curns A, Gierke R. Geographic differences in COVID-19 cases, deaths, and incidence_United States, February 12-April 7, 2020. Morb Mortal Wkly Rep. 2020;69(15):465.

40. Lieberman-Cribbin W, Tuminello S, Flores RM, Taioli E. Disparities in COVID-19 testing and positivity in New York City. Am J Prev Med. 2020;59(3):326-32.

41. Sayeed A, Kundu S, Al Banna MH, Christopher E, Hasan MT, Begum MR, et al. Mental Health Outcomes of Adults with Comorbidity and Chronic Diseases during the COVID-19 Pandemic: A Matched Case-Control Study. Psychiatr Danub. 2020;32(3-4):491-8. https://doi.org/10.24869/psyd. 2020.491

42. Mein SA. COVID-19 and Health Disparities: the Reality of "the Great Equalizer". J Gen Intern Med. 2020;35(8):2439-40. https://doi.org/10.1007/ s11606-020-05880-5.

43. Lüdecke D, von dem Knesebeck O. Protective Behavior in Course of the COVID-19 Outbreak-Survey Results From Germany. Front Public Health. 2020;8:572561. https://doi.org/10.3389/fpubh.2020.572561.

44. Mazza MG, De Lorenzo R, Conte C, Poletti S, Vai B, Bollettini I, et al. COVID19 BioB Outpatient Clinic Study group, Benedetti F. Anxiety and depression in COVID-19 survivors: Role of inflammatory and clinical predictors. Brain Behav Immun. 2020;89:594-600. https://doi.org/10.1016/j.bbi.2020. 07.037.

\section{Publisher's Note}

Springer Nature remains neutral with regard to jurisdictional claims in published maps and institutional affiliations.

Ready to submit your research? Choose BMC and benefit from:

- fast, convenient online submission

- thorough peer review by experienced researchers in your field

- rapid publication on acceptance

- support for research data, including large and complex data types

- gold Open Access which fosters wider collaboration and increased citations

- maximum visibility for your research: over $100 \mathrm{M}$ website views per year

At $\mathrm{BMC}$, research is always in progress.

Learn more biomedcentral.com/submissions 